\title{
Herschel CHESS discovery of the fossil cloud that gave birth to the Trapezium and Orion KL (Corrigendum)
}

\author{
A. López-Sepulcre ${ }^{1}$, M. Kama ${ }^{2}$, C. Ceccarelli ${ }^{1}$, C. Dominik ${ }^{2,3}$, E. Caux ${ }^{4,5}$, A. Fuente ${ }^{6}$, and T. Alonso-Albi ${ }^{6}$ \\ ${ }^{1}$ UJF-Grenoble 1/CNRS-INSU, Institut de Planétologie et d'Astrophysique de Grenoble (IPAG) UMR 5274, 38041 Grenoble, France \\ e-mail: ana.sepulcre@obs.ujf-grenoble.fr \\ 2 Astronomical Institute Anton Pannekoek, University of Amsterdam, Amsterdam, The Netherlands \\ 3 Department of Astrophysics/IMAPP, Radboud University Nijmegen, Nijmegen, The Netherlands \\ 4 Université de Toulouse, UPS-OMP, IRAP, Toulouse, France \\ 5 CNRS, IRAP, 9 Av. colonel Roche, BP 44346, 31028 Toulouse Cedex 4, France \\ ${ }^{6}$ Observatorio Astronómico Nacional, PO Box 112, 28803 Alcalá de Henares, Madrid, Spain
}

A\&A 549, A114 (2013), DOI: 10.1051/0004-6361/201219416

Key words. astrochemistry - ISM: abundances - ISM: molecules - ISM: clouds - photon-dominated region (PDR) - errata, addenda

An error occurred during the production process. The right panel of Fig. 1 was the same as the left one. The correct Fig. 1 is shown here.
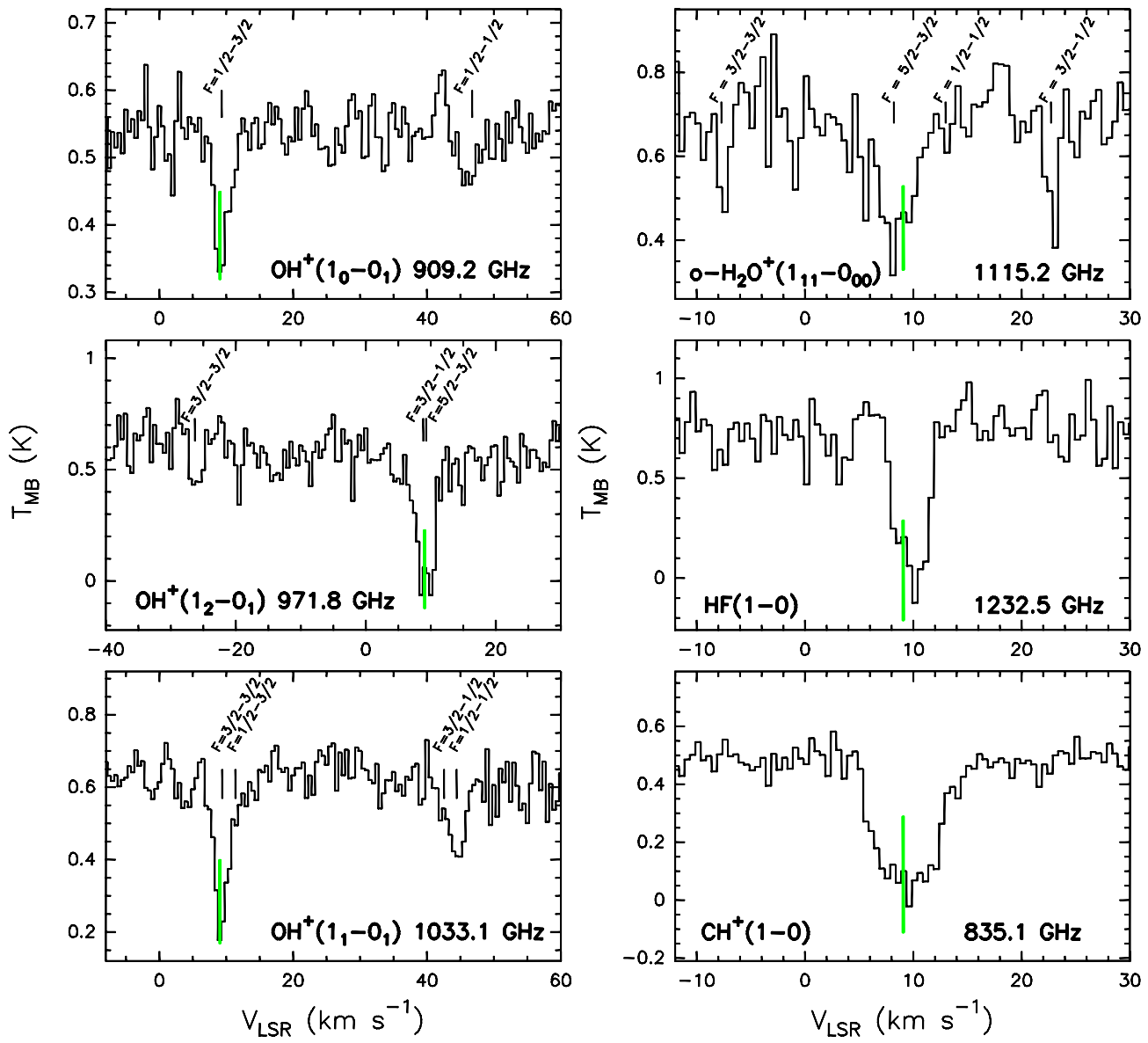

Fig. 1. Spectral lines detected in absorption towards OMC-2 FIR 4. The green vertical lines mark $V_{\mathrm{LSR}}=9.3 \mathrm{~km} \mathrm{~s}^{-1}$, i.e. the systemic velocity of the $\mathrm{OH}^{+}$lines. 